\title{
APRENDIZAJE COOPERATIVO SOBRE LA PRÁCTICA CLINICA EN ENFERMERÍA: EVALUACIÓN DE DOCENTES
}

\author{
María-Aurora Rodríguez-Borrego ${ }^{1}$, María-Jesús Movilla-Fernánez², Emma Rodríguez Maseda
}

\footnotetext{
${ }^{1}$ Doctora por la Universidad de Valladolid. Profesora de la Facultad de Enfermería de la Universidad de Córdoba. Hospital Universitario Reina Sofia. Córdoba, España. E-mail: en1robom@uco.es

${ }^{2}$ Doctora por la Universidad de A Coruña. Profesora de la Escuela de Enfermería y Fisioterapia de la Universidad de A Coruña, Campus de Ferrol, España. E-mail: movilla@cdf.udc.es

${ }^{3}$ Doctoranda de la Universidad de A Coruña. Profesora colaboradora en la Escuela de Enfermería y Fisioterapia de la Universidad de la Coruña, Campus de Ferrol, España. E-mail. emseda@udc.es
}

RESUMEN: El proyecto se desarrolló entre las Universidades de Córdoba y A Coruña en España y consistió en una experiencia de aprendizaje cooperativo intercultural e Interuniversitario con alumnos/as del primer curso de la Diplomatura de Enfermería que estaban realizando sus prácticas clínicas, con los objetivos de encontrar puntos de encuentro a la habitual distorsión entre teoría y práctica que detecta el alumnado cuando realiza sus primeras prácticas clínicas, trabajar en grupo cooperativo y ganar habilidad para la crítica y la autocrítica. Se presenta la evaluación de la experiencia por los docentes participantes con el modelo DAFO (Dificultades, Amenazas, Fortalezas y Oportunidades). Para los alumnos, el encuentro de la igualdad en la diferencia se constituyo en una gran oportunidad de aprendizaje, si bien contrastó con la dificultad de trabajar en grupo cooperativo. Como conclusión se puede decir que el método docente empleado mostró su efectividad y su dificultad.

DESCRIPTORES: Aprendizaje. Educación superior. Docentes. Enfermería.

\section{APPLICATION OF COOPERATIVE LEARNING MODEL IN CLINICAL NURSING PRACTICE: FACULTY ASSESSMENT}

\begin{abstract}
The project was developed in Spain between the University of Córdoba and the University of A Coruña. It consisted of an intercultural, interuniversity, cooperative learning experience among first year undergraduate Nursing students during their clinical practice period. The aims of this project were to find common ground in the usual distortion between theory and practice that the students detect when they develop their first clinical practice; to work in cooperative teams; and to increase critical and self-critical skills. Faculty participant experience was evaluated using the SWOT model (Strengths, Weaknesses, Opportunities and Threats). For students, finding equality among differences presented itself to be a great learning opportunity, despite the trouble derived from cooperative teamwork. In conclusion, we can affirm that the teaching method employed showed its effectiveness and complexity.
\end{abstract}

DESCRIPTORS: Learning. High education. Teacher. Nursing.

\section{APRENDIZAGEM COOPERATIVA SOBRE A PRÁTICA CLÍNICA EM ENFERMAGEM: AVALIAÇÃO DE DOCENTES}

RESUMO: O projeto se desenvolveu entre as Universidades de Córdoba e A Coruña e consistiu em uma experiência de aprendizado cooperativo, intercultural e interuniversitario, com alunos/as do primeiro ano do curso de enfermagem, que estavam realizando suas práticas clínicas. Os objetivos foram achar pontos de encontro na habitual distorção entre a teoria e a prática, que os alunos detectam no seu primeiro, contato com a prática clínica, trabalhar em grupo cooperativo e ganhar habilidade para a critica e a autocrítica. Apresenta-se a avaliação da experiência pelos docentes participantes feita com o modelo DAFO (Dificuldades, Ameaças, Fortalezas e Oportunidades). Para os alunos, o encontro da igualdade na diferença se constitui numa grande oportunidade de aprendizagem, embora se contrastou com a dificuldade de trabalhar em grupo cooperativo. Como conclusão, podemos dizer que o método docente mostrou sua efetividade e sua dificuldade.

DESCRIPTORES: Aprendizagem. Educação superior. Docentes. Enfermagem. 


\section{INTRODUCCIÓN}

El conocimiento se construye en colaboración, en interrelación y con respuestas responsables. ${ }^{1-2} \mathrm{La}$ educación democrática en el aula, se presenta como un eficaz instrumento en esa elaboración cognitiva. Una educación basada en la reflexión y la crítica es el germen fundamental para una transformación de la sociedad, al formar personas con la actitud responsable de quien se implica en la problemática de su entorno. Son principios de la educación democrática en el aula: promover el desarrollo de cada persona en cooperación con los demás; respetar la iniciativa individual, la igualdad de condiciones y la libertad intelectual; ${ }^{3}$ en resumen, fomentar la participación real y activa de los estudiantes en la construcción de su propio proceso educativo. ${ }^{2} \mathrm{La}$ posibilidad de la aplicación práctica de la educación democrática en la formación enfermera, se ha presentado de forma circunstancial, a partir del enfoque renovador del Espacio Europeo de Educación Superior (EEES); y circunstancial, porque la educación democrática, tiene sus orígenes en $1963,{ }^{4}$ mucho tiempo antes que estos aires renovadores convergentes europeos, y ni que decir tiene si nos remontamos a Sócrates, 300 años ante de nuestra era. Así como en otras experiencias docentes en otros entornos culturales y distantes de la vieja Europa como Sudáfrica ${ }^{5}$, Canada ${ }^{6}$ o Australia. ${ }^{7}$ El EEES hace hincapié en enseñar desde un nuevo paradigma docente centrado en el estudiante. Toda ésta, "en teoría", revolución paradigmática exige nuevos métodos docentes, con los objetivos claros, centrados en un mayor compromiso y protagonismo del estudiante en su formación, que ineludiblemente van unidos a la adquisición de determinadas competencias ${ }^{8}$, como la capacidad crítica y autocrítica o la toma de decisiones. En el caso específico de la formación enfermera, preparar a los discentes para responder ante lo imprevisible, precisa, necesariamente, instaurar métodos docentes que les impliquen, de modo que su proceso de aprendizaje se constituya en un proceso vital arraigado. Con esta perspectiva se puso en marcha una actividad de innovación con los siguientes objetivos: encontrar puntos de encuentro a la distorsión teoría y práctica detectada por los alumnos en sus primeras practicas clínicas, a partir del análisis de dos entornos asistenciales y culturales distintos; y trabajar en competencias genéricas como la habilidad para la crítica y auto critica, la apreciación de la diversidad, la capacidad para comunicarse con pares de otro entorno cultural y la capacidad para trabajar en equipo cooperativo.

\section{METODOLOGÍA}

Proyecto interuniversitario en la Titulación Diplomado en Enfermería, con la participación de 20 alumnos, diez de la Universidad de Córdoba, y diez de la Universidad de A Coruña; y tres profesoras, dos de la Universidad de A Coruña y una de la Universidad de Córdoba, ambas Universidades Españolas. En este manuscrito se presenta la evaluación que, de la experiencia, hicieron las docentes responsables del proyecto; si bien entendemos que es necesario explicar todo el proceso para comprender la evaluación realizada. El período de desarrollo fue del 30 de abril al 17 julio de 2009; y los criterios de inclusión: alumnos de primer curso que realizaran sus prácticas clínicas y carácter voluntario. Esta experiencia se valoro como actividad complementaria y obtuvo seis créditos de Libre Elección Curricular en la Universidad de Córdoba. Se constituyeron dos grupos de 10 miembros, con cinco personas de cada universidad. De modo que las actividades, en lo que a las profesoras tutoras se refiere, se hicieron por duplicado por cada grupo de diez alumnos. En la formación de los grupos se busco la heterogeneidad en relación al lugar donde hacian las practicas, dado que unos las realizaban en hospital, otros en centros sociosanitarios y otros de discapacitados. El procedimiento seguido fue: 1- Sesión de video conferencia para presentación y toma de contacto de los participantes y tutores, explicación de objetivos, planteamiento de plan de trabajo, abierto al consenso con los alumnos y con posibilidad de modificación en la medida que fueran evolucionando los acontecimientos; presentación de criterios de evaluación de la experiencia: Debilidades, Amenazas, Oportunidades, Fortalezas (DAFO). 2- Sesión tutorial semanal, a través de video conferencia, de dos horas de duración. En cada sesión se indicaban los temas a trabajar en método cooperativo por los alumnos, para presentar en la tutoría siguiente. Estos temas eran: dificultades de aprendizaje en el entorno clínico en cuanto al manejo de sentimientos en torno a la profesión; comportamientos ante el dolor, invalidez y manejo de enfermedades infecciosas; manejo de incertidumbre y toma de decisiones y afrontamiento de problemas éticos; estás propuestas no excluían que se trataran temas emergentes. 3- De cada sesión se hizo un informe que se colgaba en la plataforma Moodle, a la vez que profesoras y alumnos escribían su valoración personal en los diários; estas valoraciones parciales sirvieron para modificar y mejorar la actividad realizada. 4- Dos 
sesiones de video conferencia, para evaluación, al finalizar la parte on line y las estancias clínicas. De ambas se realizo informe por consenso. 5- Estancias consecutivas de uma semana, de los diez alumnos de Córdoba en A Coruña y de los diez de A Coruña en Córdoba, en los centros asistenciales donde realizaban sus prácticas. 6- Informe final consensuado. En el analisis de contenido de toda la documentación elaborada se siguieron dos vertientes: ${ }^{9}$ analisis de la narrativa: ¿cómo se cuenta? y análisis temático/categorial ¿Qué se cuenta?. En la reducción de los datos, el método facilitador fue: ${ }^{10}$ unidades de significado, enunciados de significado, temas comunes. Si bien había unas categorias de partida: Debilidades, Amenazas, Fortalezas y Oportunidades (DAFO) que son, en lo que a los profesores se refiere, a las que nos vamos a referir en este manuscrito. La triangulación en el conjunto del proyecto fue de investigadores: tres profesoras de dos ambientes culturales y universitários diferenciados, sujetos de estúdio, y técnicas de recogida de datos: observacion participante de profesores y alumnos (registrado en los informes consensuados) y diários reflexivos.

\section{RESULTADOS}

Como se ha señalado en el apartado prévio los datos que aqui se ofrecen van referidos a la evaluación realizada por las profesoras sobre la experiencia docente, teniendo como referencia los objetivos señalados y describiendo las Dificultades, las Amenzas, las Fortalezas y las Oportunidades que encontraron en la puesta en practica de la estratégia docente. Se reflejan los enunciados de signifcado construídos por las profesoras, a partir de las unidades de significado, las suyas y las de los alumnos, protagonistas ambos del proceso. Esas unidades codificadas y agrupadas según el modelo DAFO son:

Debilidades: "Los alumnos/as no están concienciados de la importancia/necesidad del trabajo cooperativo"; "Aún hay alumnos y alumnas que no han iniciado su agenda, es decir no han hecho su propia reflexión personal sobre lo acontecido, no lo han interiorizado, lo que puede llevarles, en las sesiones, a no participar con convicción, condicionando la dinámica grupal"; "Los intereses que han llevado al alumnado a participar en la experiencia"; "No todo el alumnado participante en la experiencia está del todo integrado, las aportaciones son limitadas"; "Debilidades y fortalezas van de la mano, la participación se circunscribe al más interesado o implicado o quizá al que se le haya planteado alguna situación, pero sigue habiendo grandes silencios"; "Afrontar aspectos como grupo cooperativo, autoevaluación, heteroevaluación sin conocer los conceptos, su significado".

Amenazas: "Algunas de las expectativas planteadas (ver una ciudad o conseguir unos créditos) sin estar acompañadas de una oportunidad de aprendizaje, puede ser una rémora en el desarrollo del trabajo del grupo"; "Los sentimientos aflorados surgen de experiencias vividas con intensidad, por lo que existe el peligro de quedarse en la narración de las vivencias y no ir al fondo de las mismas expresado en sentimientos y/o conductas"; "Las comparaciones entre grupos dentro de la misma sesión y entre sesiones, comparaciones que deben evitarse, estamos en un proceso de aprendizaje no en una competición"; "La riqueza cultural de la experiencia ha de servir para enriquecer no para entorpecer"; "La tecnología se ha convertido en un gran obstáculo;, "La sobrecarga emocional y de trabajo que ha supuesto la experiéncia".

Fortalezas: “La gran participación por parte de todos los miembros de los dos grupos. Quizá al segundo turno les ha costado un poco más esfuerzo pero al analizar el trabajo se observan grandes aportaciones"; "Esta participación activa esta retroalimentando la experiência";"Como contrapunto a las debilidades, hay alumnos y alumnas que reflexionando en sus diarios se van implicando más y van viendo las necesidades, las oportunidades y las debilidades y ofrecen sus sugerencias, ellos y ellas mismas señalan que se va creando un ambiente motivador y cómodo para las sesiones siguientes animando a continuar en el camino emprendido"; "A pesar de lo expresado en el apartado debilidades en la segunda sesión de hoy se ha observado un intento de mejorar la situación, dando su frutos, y así lo han expresado algunas personas es sus diários"; "Los temas de interés en la profesión enfermera, y el tema de hoy era especialmente interesante, al evidenciarse que los problemas éticos están en la cotidianeidad de la labor enfermera, ha hecho que la participación fuera más intensa"; "El esfuerzo de profesoras y alumnos/as por llevar a cabo la experiencia a pesar de las muchas dificultades tanto de infraestructura como de concepto".

Oportunidades: "Los dos entornos asistenciales diferenciados permiten contrastar fundamentalmente las semejanzas de sentimientos y de conductas"; "Esas semejanzas sirven a los grupos participantes para actuar como grupos de apoyo mutuo en el afrontamiento de las diversas viven- 
cias que comparten"; "Las dificultades tecnológicas y de comunicación intragrupo e intergrupos se muestran como grandes oportunidades para la mejora, llevando a la reflexión sobre lo realizado hasta ahora, permitiendo mejorar el planteamiento metodológíco de la experiencia y animando a modificación de las actitudes de los participantes en el convencimiento de que es un aprendizaje cooperativo donde todos colaboran, todos se enriquecen"; "Dado que la experiencia docente se enmarca dentro del marco de conocimiento de los cuidados básicos, las experiencias compartidas de los centros sociosanitarios se están presentando como valiosos instrumentos de aprendizaje en este sentido"; "Constatar que en entornos asistenciales y culturales distintos, las incertidumbres que viven los alumnos y alumnas de Enfermería tienen un foco u origen común"; "La igualdad en la diferencia hace plantear estrategias comunes que permitan avanzar"; "La propia sesión conjunta e intercultural que permitió sacar el sentimiento real de los alumnos ante las metodologías participativas, cooperativas; sus temores y rechazo ante las mismas. Planteadas en el entorno evaluador de la experiencia vivida há hecho que las exposiciones que previamente habían expuesto resultaran contradictorias. Por lo que finalmente se puso sobre la mesa la evaluación real".

\section{DISCUSIÓN}

El entorno metodológico de la investigación cualitativa, hubiera permitido interrelacionar el apartado resultados y discusión del manuscrito, en el análisis de los enunciados de significado con las categorias de partida, y relacionarlo con la literatura existente al respecto. Pero el método empleado ${ }^{3}$ en la reducción de los datos, tiene como primer paso el de "lectura de las notas recogidas para obtener un sentido global". Y es ese sentido global el que nos gustaría que se adquiriera sobre la experiencia; ya que uno de los aspectos interesantes del método cualitativo es el de permitir al lector hacer su personal interpretación y elaboración de la información que recibe e interactuar con ella. Las profesoras, sujetos participantes de la experiencia docente, reflejan en sus aportaciones como los objetivos se fueron consiguiendo paulatinamente sobre un marco común, que se definio desde el inicio, y que fue el de la igualdad en la diferencia, ${ }^{11}$ los alumnos fueron por tanto consiguiendo el objetivo de apreciar la diversidad a partir del encuentro con la semejanza de sentimientos, actitudes y comportamientos en todos ellos, respecto a la profesión elegida y respecto a los profesionales asistenciales de los centros donde desarrollaban sus practicas; fondo de trabajo de las tutorias; poniendo en evidencia la importancia de la refexión de la practica y sobre la practica; ${ }^{12}$ a la vez que el valor del aprendizaje interpares. ${ }^{13}$ Se vislumbró una dificultad, también común, para la capacidad crítica y la autocrítica, a la que finalmente se llegó al constatar que se están poniendo en práctica técnicas basadas en conceptos aún no aprendidos, entendidos, vivenciados; "Students must be led gently into the active role of discussing, dialoguing, and problem solving". ${ }^{14: 461} \mathrm{La}$ dificultad de trabajar en grupo cooperativo quizá relacionada con la dificultad de comunicación y los intereses personales. ${ }^{2}$ Un aspecto a destacar, por la gran contradicción que lleva implícito, es el de las nuevas tecnologías; son numerosas las experiencias docentes que, desde hace tiempo, apoyan su uso en la docencia ${ }^{15-16}$, si bien en esta experiencia fue un arma de doble filo en la medida en que a la vez que eran el centro de la actividad, permitiendo que se llevara a cabo, los fallos de conexion se erigian como amenazas permanentes. Un aspecto que se codifico como amenaza fue la sobrecarga emocional y de trabajo que supuso todo el proceso, al ser una actividad añadida al resto de trabajo curricular, pero que se contrarresto con la fortaleza del esfuerzo de profesoras y alumnos por llevar a cabo la experiencia a pesar de las muchas dificultades tanto de infraestructura como de concepto; se puede deducir pues que las fortalezas y las oportunidades brindaban benefícios. ${ }^{7}$

\section{CONCLUSIONES}

Los objetivos del proyecto se alcanzaron al propiciar las circunstancias que favorecían su consecución, todos ellos contribuciones especificas para la formación de los enfermeros; lo que resalta la potencialidad del aprendizaje cooperativo, sin embargo hay aspectos que se muestran contadictorios, lo positivo del aprendizaje adquirido contrasta con las dificultades del trabajo en grupo cooperativo y el dialogo entre ambos grupos; presentando al "grupo" como instrumento y como dificultad. Lo que hace que, siendo esta una actividad voluntária, de forma genérica y en relación al proceso de aprendizaje a través de la educación democrática, las palabras de Freire cobren especial sentido, cuando señala que la decisión de asumir las consecuencias del acto de decidir forman parte del aprendizaje o que "educar não é a mera transferência de conhe- 
cimentos, mas sim conscientização e testemunho de vida, senão não terá eficácia". ${ }^{11: 21}$

Se puede decir, por tanto, que el método docente empleado mostró su efectividad y su dificultad; y que los cambios o ajustes posibles, para la mejora del modelo, pasan por persistir en su puesta en práctica, dado que las dificultades radican en el propio ser humano y en las dificultades de interacción con sus pares, "el aprendizaje de los alumnos en el enfrentamiento con la realidad orientado por la investigación pedagógica". 17:120

\section{REFERENCIAS}

1. Johnson D, Johnson R. El aprendizaje cooperativo en el aula. Barcelona (ES): Paidós, 1999.

2. Rodríguez Borrego MA, Boronat Mundina J, Freire I. Metodologias colaborativas, educação na e para a responsabilidade na formação em enfermagem. Sísifo. 2008; 7:6374. [Acceso 2010 Jun 8] Disponible em: http:/ / sisifo.fpce.ul.pt/? $r=19 \& p=63$

3. Romo AP, Nubiola J. Virtudes democráticas en la educación de hoy: la propuesta pedagógica de John Dewey. 2005. [acceso 2010 Jun 8] Disponible en: http://www.unav.es/gep/Dewey/ PropuestaPedagogicaRomoNubiola.html

4. Dewey J. Democracia y educación. Buenos Aires (AR): Losada, 1971.

5. Mangena A, Chabeli MM. Strategies to overcome obstacles in the facilitation of critical thinking in nursing education. Nurse Educ Today. 2005 May [acceso 2010 Jun 8]; 25:2918. Disponible en: http:/ / www.sciencedirect.com/science?_ob=MImg\&_ imagekey=B6WNX-4FXNR91-1-1\&_cdi=6974\&_ user $=687353 \&$ \& pii $=$ S0260691705000286\&_ origin $=$ search\&_zone =rslt_list_item \&_ c o ver D a te $=05 \% 2$ F $31 \% 2$ F 2005 \& sk $=999749995 \& w c h p=d G L z V z z-z S k z V \& m d 5=c 27$ dc7cf13bb12e35921b8dd8d303fe3\&ie=/sdarticle.pdf

6. Epp S. The value of reflective journaling in undergraduate nursing education: a literature review. Int J Nurs Stud. 2008 Sep [acceso 2010 Jun 8]; 45(9):137988. Disponible en: http:// www.sciencedirect.com/science?_ob=MImg\&_ imagekey $=$ B6T7T-4S08YP9-1-1\&_cdi $=5067 \&$ _ user $=687353 \&$ _pii $=$ S0020748908000266\&_ origin=search\&_zone=rslt_list_item\&_ c ove r D a t e $=09 \% 2$ F $30 \% 2$ F 2008 \& sk $=999549990 \& w c h p=d G L z V l z-z S k z S \& m d 5=c 561$ 3549d0bf25094e110bdd1fc12050\&ie=/sdarticle.pdf

7. Eps MA, Cooke M, Creedy D, Walker R. Student evaluations of a year long mentorship program: a quality improvement initiative. Nurse Educ Today. 2006 Ago [acceso 2010 Jun 8]; 26(6):51924. Disponible en: http://www. sciencedirect.com / science?_ob=MImg\&_ imagekey=B6WNX-4JGJHWB-2-1\&_cdi $=6974 \&_{-}$ user $=687353 \&$ \& pii $=$ S0260691706000104\&_ origin=search\&_zone=rslt_list_item \& c o ver D a te $=08 \% 2$ F $31 \% 2$ F 2006 \& sk=999739993\&wchp=dGLzVlz-zSkzS\&md5=09af cf490fb128837830b84bf7cbea3c\&ie=/sdarticle.pdf

8. González J, Wagenaar R, coodenadores. Tuning educational structures in Europe. informe final. fase uno. Bilbao (ES): Universidad de Deusto, 2003.

9. Gerrish K, Lacey A. Investigación en enfermería. Madrid (ES): McgrawHill/ Interamericana de España; 2008.

10. Solis Muñoz M, Calvo Cilla P, García López F, Iglesias López MA, Pérez-García R, Corona-Muñoz. Percepción de la calidad asistencial en mujeres a las que se les ha realizado una mamografía. Metas Enferm. Abr 2009; 11(3):149

11. Freire P. Pedagogía de la autonomía. São Paulo: siglo XXI, 1997.

12. Hallet CE. Learning through reflection in the community: the relevance of Schon's theories of coaching to nursing education. Int J Nurs Stud. Abr 1997; 34(2):10310. [acceso 2010 Jun 8] Disponible en: http://www.sciencedirect.com/science? ob=ArticleURL\&_udi=B6T7T-3RGSW9K3\&_ user $=10 \&$ _coverDate $=04 \% 2$ F30 $\% 2$ F $1997 \&$ _ alid $=1720268973 \& \_r d o c=1 \& \_f m t=h i g h \&$ orig $=$ search\&_origin $=$ search\&_zone $=$ rslt_list_ item\&_cdi $=5067 \&$ sort $=r \& \_s t=0 \&$ docanchor $=\&$ _ $c t=1 \&$ \& act $=C 000050221 \&$ _version $=1 \&$ _ urlVersion $=0 \& \_$userid $=10 \& \mathrm{md} 5=$ f $997 \mathrm{fa} 883$ ade 683 76b663f2553ace752\&searchtype $=a$

13. Yuen AY, Chow F L W. Learning partnershipthe experience of peer tutoring among nursing students: a qualitative study. Int J Nurs Stud. Feb 2007; 44: 23744. [acceso 2010 Jun 8] Disponible en http://www.sciencedirect.com/ science?_ob=ArticleURL\&_udi=B6T7T-4J2KY0K $5 \&$ _user $=10 \&$ \&coverDate $=02 \% 2 \mathrm{~F} 28 \% 2 \mathrm{~F} 2007 \&$ alid $=1720268230 \& \_r d o c=1 \&$ fmt $=$ high \&_ orig $=$ search\&_origin=search\&_zone $=$ rslt_list item\&_cdi $=5067 \& \_s o r t=d \& \_s t=0 \& \_d o c a n c h o r=\& \_$ $c t=1 \& \_a c c t=C 000050221 \&$ _version $=1 \&$ urlVersion $=0 \&$ userid $=10 \& m d 5=4152$ acf 3 ad 2537 d5e0f0ca9dec553936\&searchtype $=a$

14. Myrick F, Yonge OJ. Crating a climate for critical thinking in the preceptorship experience. Nurse Educ Today. Ago 2001;21:4617 [acceso 2010Jun8]. Disponible en: http://www.sciencedirect.com/science? $o b=$ ArticleURL\&_udi=B6WNX-45V298C-6\&_ user $=10 \&$ _coverDate $=08 \% 2 \mathrm{~F} 31 \% 2 \mathrm{~F} 2001 \&$ _ alid $=1720265516 \& \_r d o c=1 \& \_f m t=h i g h \&$. orig $=$ search \&_origin $=$ search\&_zone $=$ rslt_list_ item\&_cdi $=6974 \&$ sort $=r \& \_s t=0 \&$ docanchor $=\&$ _ $c t=1 \& \_$acct $=C 000050221 \& \_$version $=1 \&$ urlVersion $=0 \& \_$userid $=10 \& \mathrm{md} 5=6341 \mathrm{fa} 3 \mathrm{c} 3 \mathrm{f} 7373 \mathrm{~b} 5$ 27f6d2a7dd5d1e6c\&searchtype $=\mathrm{a}$

15. Fernández Díaz A, Correa Gorospe JM. La integración educativa de las tecnologias de la 
información y comunicación: una experiencia de acción formativa para reconstruir el curriculum investigando sobre la propia practica. Current Developments in TechnologyAmsted Education. 2006 [acceso 2010 Jun 8]. Disponible en: http:// www.portaleducativo.hn/pdf/523527.pdf

16. Vizcaya Moreno MF, Romá Ferri MT. Uso de las nuevas tecnologias en la formación de profesionales de enfermería. Metas de Enferm. 2000 May; 25: 258

17. Maeda, ST, Chiesa A. Innovación en el proceso de aprendizaje de enfermería en salud colectiva. Texto Contexto Enferm. 2010 Abr-Jun [acceso 2011 Abr 17]; 19(2):120-8. Disponible en: http://www. scielo.br/scielo.php?script=sci_arttext\&pid=S010407072010000100014\&lng=pt\&nrm=iso 